\title{
Using mobile technology to improve maternal, child and youth health and treatment of HIV patients
}

\section{Case study: Stock-out of iron} supplements in pregnancy

In February 2015, the South African (SA) National Department of Health (NDoH) was notified of a shortage of iron tablets at a clinic in Kenneth Kaunda District in North West Province. Similar reports emerged from two other clinics in the same district around the same time. Iron supplements are routinely provided to pregnant women in SA to prevent maternal anaemia. Maternal anaemia is a risk factor for mortality from obstetric haemorrhage, which accounts for more than 200 maternal deaths annually. ${ }^{[1]}$

These reports resulted in a broader enquiry encompassing the whole of the province, revealing widespread stock-outs. With this insight, the provincial Department of Health met with staff to re-emphasise ordering procedures and what to do if drugs are out of stock.

Notably, the earliest reports of iron stock-outs were received not from clinic staff but from pregnant women themselves, using the help desk function of a new mobile phone-based system called MomConnect.

\section{MomConnect}

This case study illustrates the potential of mobile health (mHealth) to improve the health system. MomConnect ${ }^{[2]}$ was the first, HIV treatment guidelines the second, and B-Wise the third of a series of $\mathrm{NDoH}$ initiatives, launched by the Minister of Health, that use mobile technology. These uses include engaging with users and providers of health services, providing accessible health information, and capturing feedback on the quality of care.

\section{The highest cellphone penetration in Africa}

These initiatives take advantage of SA's extremely high rate of mobile telephone use. According to a recent Pew Research Center report, $90 \%$ of South Africans own a cell phone. ${ }^{[3]}$ In addition, we have the highest smartphone ownership on the continent $(34 \%)$ and are the only African country among those surveyed in the Pew report that did not have a gender gap in phone ownership, with equal numbers of males and females reporting cell phone ownership and use.

\section{What is Mom Connect?}

MomConnect was conceptualised with four components, which are at various stages of implementation:

1. A USSD-based registration system to enroll every pregnant woman in the country into a national pregnancy register, using her ID number as a unique identifier. (Unstructured Supplementary Service Data (USSD) is a text-based system that works on even the most basic cell phone. In SA, a majority of cell phone users regularly use USSD for airtime top-ups and checking airtime balances.) This is seen as one of the elements of the macro plan towards adoption of a national electronic medical record.

2. Weekly text messages with information on healthy pregnancy and care of a newborn, timed according to estimated date of delivery and later the age of the infant, are sent to the registered woman.

3. A help desk to allow women to ask additional questions, submit compliments or complaints, or rate the quality of care received.

4. A supportive and empowering SMS (text message) and mobile web component for midwives and nurses that will provide them with clinical updates and enable them to interact with each other as well as give feedback to managers.

The programme was launched by Minister of Health Aaron Motsoaledi in August 2014, and registered over 500000 women in its first year. In the same timeframe, more than 34000 health workers were trained to register mothers on MomConnect in 95\% of public health facilities. Over 3000 mothers complimented the services and 500 complained, a ratio of six compliments to every complaint. More than 180000 questions were answered through the help desk. All patient feedback captured via MomConnect is clinicspecific (each clinic has been assigned a unique code that is captured during patient registration), and is routed to the clinic in question via assigned district focal persons. This provides a near real-time view of service delivery quality around the country, allowing rapid detection of remediable problems such as the iron shortages described above.

\section{How does Mom Connect work?}

Women who suspect they may be pregnant are able to self-subscribe (by dialling ${ }^{\star} 134^{\star} 550 \#$ ) for a limited set of text messages that provide information on the MomConnect service and the importance of receiving antenatal care as early as possible. These messages encourage women to go to an antenatal clinic.

Once a pregnancy has been confirmed by a health worker at the first antenatal visit, the woman can opt to be formally registered using her ID number or her passport number as an identifier, and including a clinic code to associate her registration with the particular health facility where she is receiving care. Each registered woman receives two SMS messages a week throughout her pregnancy and for the first year of her child's life. These messages are timed to correspond with the woman's stage of pregnancy and the age of her child. Messages cover priority topics such as antenatal nutrition, testing for HIV, warning signs of maternal hypertension, preparation for labour, exclusive breastfeeding, postpartum depression and the immunisation schedule. They also include information on patient rights and responsibilities and how to provide feedback on the quality of care received.

Mothers are able to reply to any text message or ask questions by phoning ${ }^{\star} 134^{\star} 550 \#$. The help desk is currently staffed by a professional nurse who deals with complicated issues and is assisted by two clerical workers who deal with routine questions to which they pull automated answers from a suite of possible responses. Women can opt out of the service at any time by phoning ${ }^{\star} 134^{\star} 550^{\star} 1 \#$. At around the due date of delivery the messages automatically switch to the infant messaging.

As MomConnect targets all women, including those at the low end of the socioeconomic spectrum, all these services are provided free of charge to the mother.

\section{The evidence for mobile messaging to mothers}

MomConnect builds upon a growing body of work around using mobile phones to support improvements in maternal and child health. Programmes such as Aponjon in Bangladesh, Wazazi Nipendeni in Tanzania, Chipatala Cha Pa Foni in Malawi, Text4Baby in the USA and MAMA in SA (a precursor to MomConnect) have all provided regular SMS and/or voice-based messages to pregnant women and new mothers to support the adoption of healthy behaviours and increase the uptake of health services. Impact evaluations in 
Malawi and Bangladesh have demonstrated statistically significant improvements in behaviours such as early breastfeeding, use of a bed net and antenatal attendance. ${ }^{[4,5]}$

Operational research on MomConnect suggests overwhelming support by pregnant women. In a sample of nearly 10000 women telephonically surveyed, with a response rate of over one in five ( $n=1999)$, $98 \%$ found the messages helpful, 77\% felt better prepared for delivery, $81 \%$ shared their messages with family and friends, and $70 \%$ wanted more messages per week ( $\mathrm{M}$ van den Heuvel, market research report on experience of mothers using MomConnect (Johannesburg, Praekelt Foundation, October 2015) - unpublished). However, MomConnect is unique in that it provides two-way interaction rather than simple one-way messaging. By providing channels for patient feedback, it generates useful information to inform and strengthen the quality of health services. For example, in its first year MomConnect received 78916 completed surveys ranking the quality of the first antenatal visit according to four criteria: cleanliness, staff friendliness, privacy and wait times. The help desk also receives specific compliments and complaints.

Naturally the complaints get more attention from managers than do the compliments, so in addition to improving the supply side from this feedback mechanism, the $\mathrm{NDoH}$ is implementing a support mechanism to empower frontline nurses and midwives. They will be registered with their personal cell phones on MomConnect and will receive regular information to increase knowledge of guidelines and support adherence to protocols. A mobile-based learning portal will allow health workers to complete in-service training modules and form peer-to-peer learning and support networks.

\section{HIV treatment guidelines on a mobile application}

On the sidelines of the SA AIDS Conference in June 2015, the Minister of Health launched the first-ever mobile application to make HIV treatment guidelines available on a mobile phone to clinicians. The app was developed in partnership with the private sector and includes a range of functions. Besides the guidelines for treatment of HIV in children and adults, the app provides information on every antiretroviral drug that is part of the national guidelines and their side-effects, contraindications and possible adverse events. In addition, the app assists clinicians to calculate paediatric dosages and report medicine stock-outs.

Clinicians who use either an iPhone or an Android phone can download the app through either the Apple iStore or Google Play store. The app does not require internet access once downloaded. Revisions of the guideline are downloaded automatically when the phone is connected to the internet. This means that clinicians have the latest clinical guidelines literally at their fingertips.

The NDoH is in the process of developing (with the same private sector company) a similar app for the treatment of drug-susceptible as well as drug-resistant tuberculosis.

\section{Extending health information to the youth: B-Wise}

The Minister of Health launched a mobile website named B-Wise (accessible at b-wise.mobi) in August 2015 to specifically address challenges faced by the youth of SA. These health risks include bullying, obesity, unwanted and unplanned pregnancy, and being infected with HIV. B-Wise provides information in easily accessible language, gives practical information about where to get support, and will soon have a more interactive chat facility where experts on various topics will interact with the youth. In the first month after the launch, 20927 users logged onto B-Wise and 40580 pages were accessed (Jesse Coleman, Wits Reproductive Health and HIV Institute - personal communication).

\section{Vision for the future}

Technology creates the potential to match demand for health services with supply, and to create linkages between individuals, communities, frontline health workers, health facilities, laboratories and health management. A connected health system is efficient, responsive and patient centred.

MomConnect, the HIV treatment guidelines app and B-Wise are existing examples of the role of technology in linking patients and communities with health workers and the health system, and increasing access to guidelines for health workers. This work serves as an important foundation that can be built upon to reach new audiences.

Processes are in place to extend MomConnect to provide additional support to HIV-positive mothers and partners and to lengthen the period of messaging until children reach age 5 . MomConnect is also being extended to the private sector, as there is anecdotal evidence that many women first book with private general practitioners but then deliver in public health facilities. Private practitioners wishing to learn more about MomConnect and ways to engage can contact MomConnect programme manager Antonio Fernandes (fernaa@ health.gov.za).

These efforts will strengthen the continuum of care between reproductive, maternal, newborn, child and youth health, while facilitating the development of high-quality, accountable health services.

\section{Joanne Peter}

Advisor to Johnson \& Johnson Worldwide

Corporate Contributions, Digital Health

Portfolio, Cape Town, South Africa

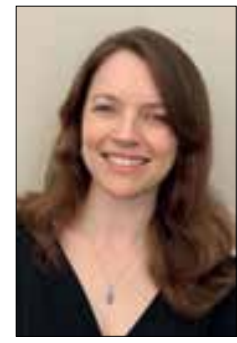

\section{Peter Barron}

School of Public Health, Faculty of Health Sciences, University of the Witwatersrand, Johannesburg, South Africa

pbarron@iafrica.com

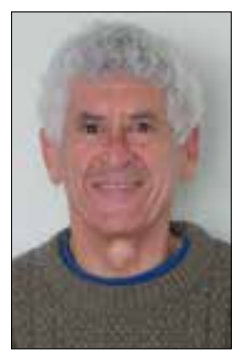

\section{Yogan Pillay}

National Department of Health, Pretoria, South Africa

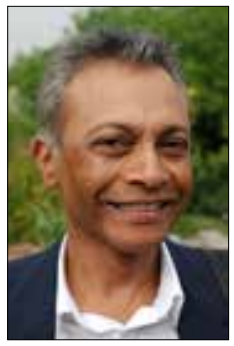

1. National Committee for Confidential Enquiry into Maternal Deaths. Saving Mothers 2011-2013: Sixth report on the Confidential Enquiries into Maternal Deaths in South Africa. Department of Health, 2014 Bateman C. Using basic technology - and corporate social responsibility - to save lives. S Afr Med J 2014;104(12):839-840. [http://dx.doi.org/10.7196/SAMJ.9118]

3. Pew Research Center. Cell Phones in Africa: Communication Lifeline. Washington, DC, Pew Research Center, 2015 http://www.pewglobal.org/2015/04/15/cell-phones-in-africa-communication-lifeline/ (accessed 15 October 2015).

4. Watkins, SC, Robinson, A, Dalious, M. Evaluation of the Information and Communications Technology for Maternal, Newborn and Child Health Project Known locally as "Chipatala Cha Pa Foni' (Health Center by Phone). Balaka District, Malawi: Invest in Knowledge Initiative, 2013

5. Chowdhury, ME. Key Findings of MAMA Study, TRAction-supported study conducted by the International Center for Diarrheal Disease Research, Bangladesh. Dhaka, Bangladesh, icddr,b, 2015 http://www.tractionproject.org/resources/key-findings-mama-study-traction-supprted-studyconducted-international-center-diarrheal (accessed 17 November 2015). 